\title{
Phenotypic and Genomic in Organ Matching in Fetal Species
}

\section{Iman Mostafa}

Islamic Shiraz University, School of Veterinary Medicine, Shiraz, Iran

Correspondence Author: 1Iman Mostafa. Islamic Shiraz University, School of Veterinary Medicine, Shiraz, Iran

Received date: 12 May 2019, Accepted date: 24 June 2019, Online date: 28 June 2019

Copyright: (C) 2019 Iman Mostafa. This is an open-access article distributed under the terms of the Creative Commons Attribution License, which permits unrestricted use, distribution, and reproduction in any medium, provided the original author and source are credited.

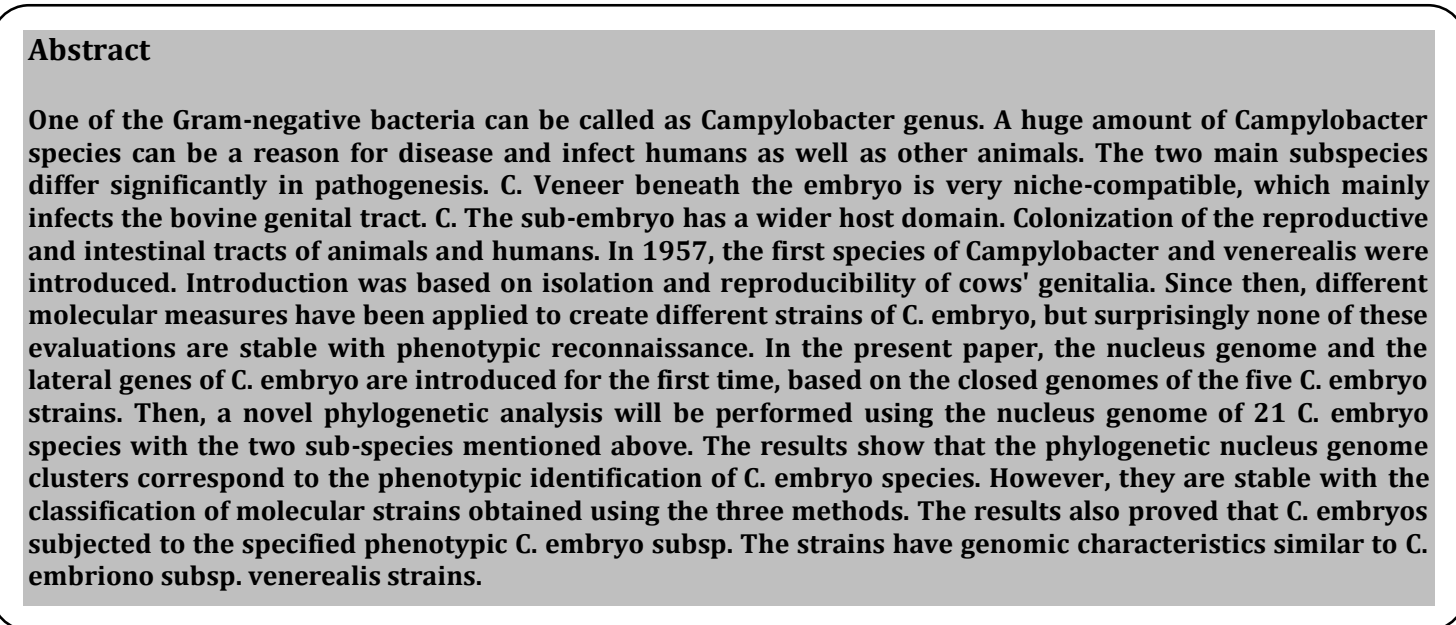

Keywords: Accounting information, Managerial work, Managers, Management Accounting Systems

\section{INTRODUCTION}

One of the most common causes of food poisoning in the world today is Campylobacter. Campylobacter species mostly become visible as curved or commashaped rods and are mighty to move through the polar or flagella. They usually outlive even in low oxygen environments. When exposed to atmospheric oxygen, the embryo C. is capable of deforming the cochlea [1]. They are visible by oxidase test and catalase test. Campylobacter spp. are inactive, and the best temperature for them is at $42{ }^{\circ} \mathrm{C}$. Campylobacteriosis is a zoonosis which is a disease as transmitted from animals to animals. Most often, carcasses or meat are infected by Campylobacter from feces at slaughter. In animals, Campylobacter rarely is a reason of disease. It is generally believed that the main route of transmission is through unprocessed meat and meat products also the raw or infected milk. Contaminated water or ice can also cause infection. During recreational activities, a number of cases occur following exposure to contaminated water. In humans, tissue injury sites include the jejunum, ileum, and colon. Figure 1 and Figure 2 , respectively, show the visible number of total food poisoning and anisakis food poisoning events prepared by the Japanese Ministry of Food Toxicity of the Ministry of Health, Labor and Welfare and the global distribution of current food poisonings [2-3]. Figure 3 shows common sources of food poisoning, which include estimated total degradation disease that can be assigned to a pathogen and a food category.

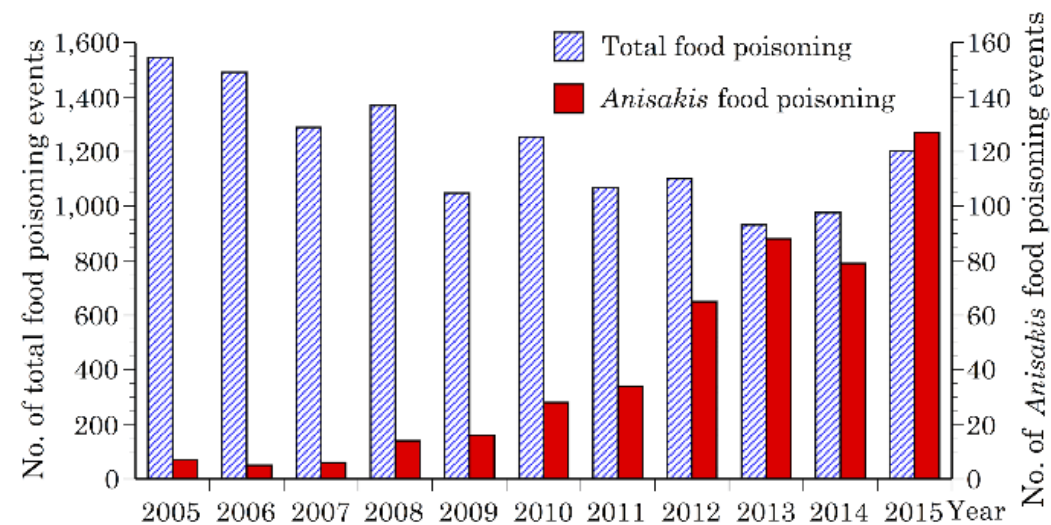

Journal of Applied Sciences Research

ISSN: 1819-544X, EISSN: 1816-157X 
Fig. 1 Reported Number of Total Food Poisoning Events and Anisakis. Food Poisoning Events Japan Statistics Food Poisoning Ministry of Health, Labor and Welfare

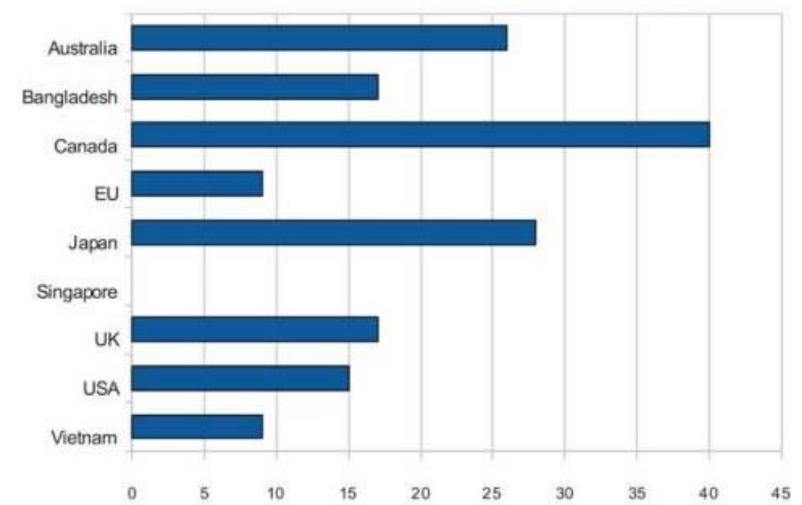

Fig. 2iFoodiSafety, NumberiofiCasesiofiFoodiPoisoningiperiYeariasiPercentageiofiPopulationiforiSomeiCountryiarounditheiWorld

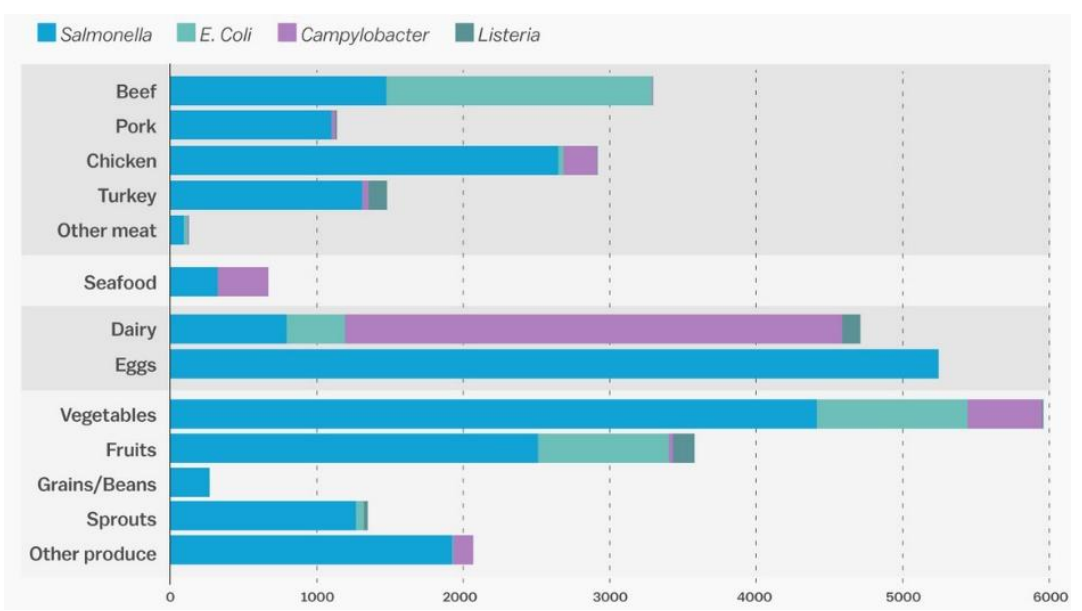

Fig. 3 Common Sources of Food Poisoning which Includes Estimated Total Illness for only Outbreaks that could be attributed to a Single Pathogen and Food Category.

Most $C$ jejuni strains produce cytolethal toxins that block cell division and prevent the immune system from activating. It helps bacteria break down the immune system and survive in the cells of the gut for a limited time. There was also a cholera-like enterotoxin believed to be produced at one time, but it seems not. Organism causes scattered, bloody, edematous and exudative enteritis. Campylobacter has been criticized in rare cases for thrombotic uremic hemolytic syndrome and thrombocytopenic purpura, although there are no reports of uncertain cases. In some cases, Campylobacter transmission can be the leading cause of GuillainBarrow syndrome. Gastrointestinal perforation is a rare complication of ideal transmission. Generally, no treatment is required, except electrolyte replacement and re-watering. Antimicrobial treatment in aggressive cases (when bacteria invade the intestinal mucosal cells and damage the tissues) or eliminate the carrier state (the condition of humans who have Campylobacter in their body and recommend that they kill the bacteria Shed while left unchecked) is recommended. Appropriate molecular kinds of methods for typing members of C. group Embryo are described.

These accounts for MLST typing techniques [2-4] and a milk typing method [1-5]. During the formation of these typing techniques, was identified the additional species might be present in the C. Embryo group. MLST analysis identified two strains, RM6137 and NCTC13003 (ST-4 and ST-1, respectively, in Figure 2 from [5]), which were closely related, but separately, to C. lanienae. RM6137 was isolated from wild pigs in California. NCTC 13003 was deposited as C. lanienae, but no epidemiological information was available for this strain.

C. Embryo Specifications

Campylobacter may be one of the 23 species currently classified in other genus. It is an aerobic, gram-negative, spiral bacterium that grows between $24.5^{\circ} \mathrm{C}$ and $37.5^{\circ} \mathrm{C}$. Unlike the exothermic C. jejuni and C. coli, not all C. embryo isolates grow at $41.5^{\circ} \mathrm{C}$. Campylobacter embryos comprise two subspecies: C. fetal embryonic species and embryonic species C. subspecies venerealis, which include biovar intermediates [6]. The subspecies are basically close together but have different habitats.

To this date, C. embryo has been recognized as an infectious animal agent. The first reservoir of C. embriono subsps is the digestive tract of cattle and sheep. However, this subspecies can also be isolated from the faeces of other animal species [2-4, 6-9]. At the same time, the natural niche of C. embriono subsp venerealis is the bovine genital tract, in which it can infect the animals themselves, showing in infertility or miscarriage [7]. A recently proposed subspecies, C. embriono subsp testudinum, which has a particular association with reptiles, has also been isolated from human disease, but has not been addressed by many researchers. The first infection of C. embryo that resulted in abortion was observed in [5].

In 1967, in the first systematic study, 21 cases of Campylobacteriosis made the distinction between Vibrio embryos (now called C. embryos) the possibility of systemic disease and "Vibrio related" disease (now known as C. jejuni and C. difficile). Coli is known to cause diarrhea [7,9]. The clinical symptoms of human C. difficile infection vary from acute diarrhea to systemic disease (Poele EM, Bolhuis H, Dijkhuizen L, 2008; Ryan A, Lynch M, Smith SM, Amu S, Nel HJ, et al., 2011, Skirrow MB, Blaser MJ, 2000). The second presentation differs from the published pathogen localization. Septicemia, with fever but without localized infection, has been reported in $24 \%-41 \%$ [2, 5, 8]. Other manifestations might be shown the nerve infections (meningitis, meningoencephalitis, subcutaneous ampoules or brain abscesses), osteoporosis, lung abscesses, osteoarthritis and perinatal infections (eg, uterine infection, abortion, or placenta) (Poele EM) [8] Campylobacter embryo infections may also the results of vascular pathology (mycotic aneurysm, endocarditis, vasculitis, thrombophlebitis, or pericarditis).

\section{RESEARCH METHODOLOGIES}

C. embryonic species will grow on heart-infused agar with 5\% goat blood over five nights in aerobic microbial conditions (5\% O2, 8\% CO2, 6\% $\mathrm{H} 2,81 \% \mathrm{~N} 2)$. Subspecies is characterized phenotypically using glycine endurance test of $0.8 \%$ and hydrogen sulfide (H2S) production, as shown [18] Molecular classification will be performed using MLST [10-12] and AFLP analysis [13, 19-20]. Perl paths have been applied for GS-Titanium sequencing of the genome data of 21 species of embryos. To confirm the assembly accuracy of AfIII and EagI high-resolution restriction enzymes. With the frequent overlap of restriction maps of unique molecules (over 50-fold overlap), the OpGen assembler program reconstructed genome restriction maps arranged from genome restriction maps were applied by 
optical generals. The alignment map for all +21 cases and the identification of the Miss assemblies allow the generation of PCR products to cover all residual gaps in the results.

\section{Analysis of Phylogenetic}

The five existing genomes for embryo are123 which were used as reference: strain 82-40 (GenBank accession number CP000487), strain 84-123 (GenBank accession number HG004426-HG004427) and strain 03/293 (accession numbers GenBank CP006999 - CP007002). Amino acid sequences of open reading frames (ORFs) encoded by seven genomes (four reference genomes plus three other genomes in this work (55/04, 38/06 and 06/386 and 97/608)) as inputs for all used. All the results are using BLASTp (-e 0.0003, 80\% cut-off similarity). ORFs present in each of the seven strains (> 80\% identity greater than $80 \%$ protein length) were part of the C. embryo nucleus genome. The strain ORF sequence 76-43 was used as the reference sequence of the major genes. Areas where SAP coding, genomic islands and restriction of correction systems, predictions and interpolation sequences were conceived as accessory genes. Accessory genes were identified in the Roche 454 enclosure of $21 \mathrm{C}$. embryo es by a BLASTn localization analysis (-e $0.0002,80 \%$ similarity cutoff) against identified accessory genes from six C. fetal strains. If the BLASTn race was> $80 \%$ greater than $80 \%$ area, the species was considered positive for specific accessory areas. Phylogenetic analysis of the nucleus genome was obtained as follows: The nucleotide sequences of the predicted genes from the Roche 454 links were generated using GeneMark (v2.8). For each nucleus gene, the nucleotide-related consequences of each strain were extracted and aligned using the gene-based MUSCLE [13-18]. The alignments were a continuous consequence for each C. embryo. Therefore, a phylogenetic maximum likelihood tree was constructed using RAxML (v7.2.8) under the GTRCAT method.

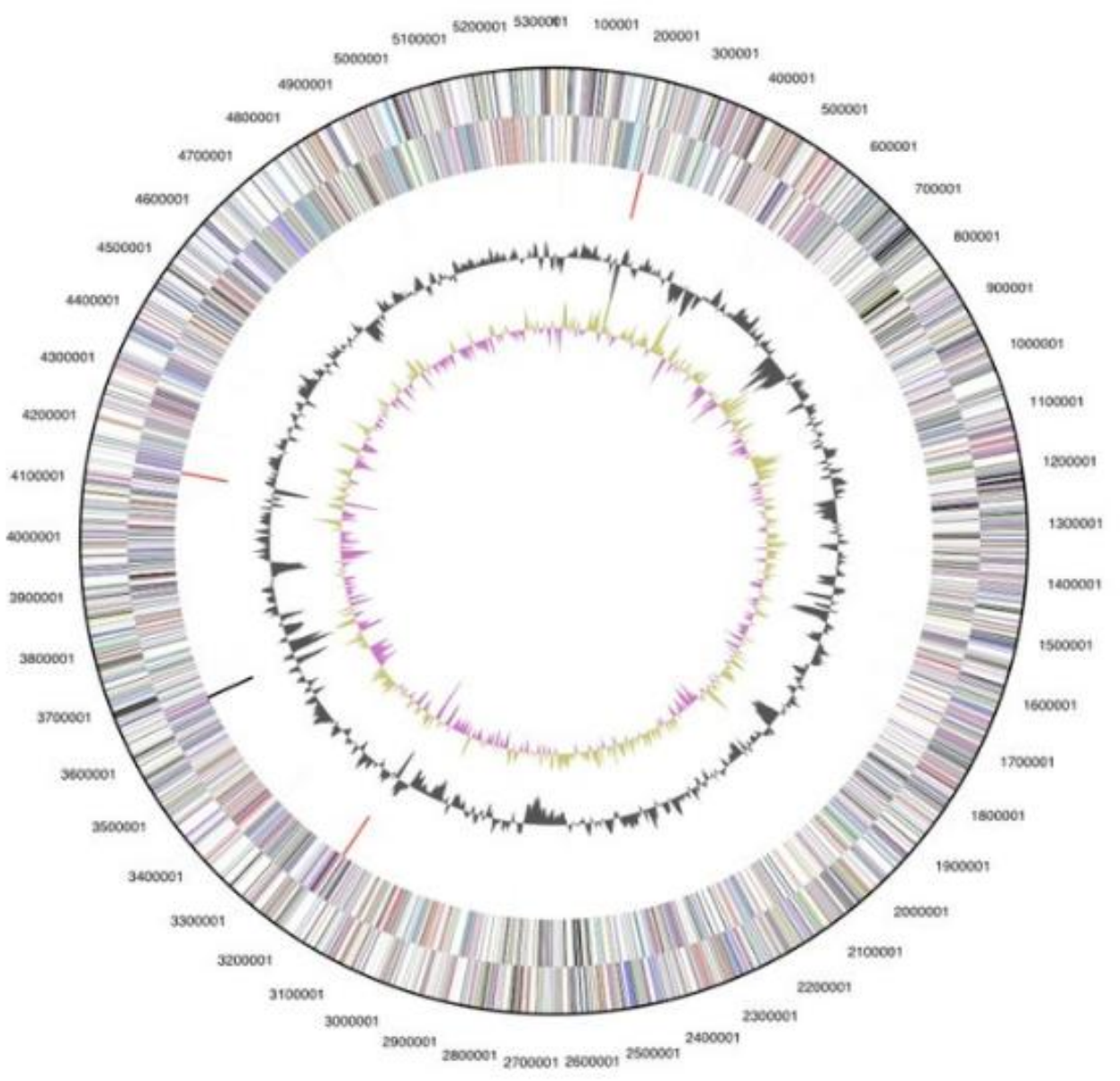

Fig. 4 Graphical Circular Map of the Genome from Outside to the Center 


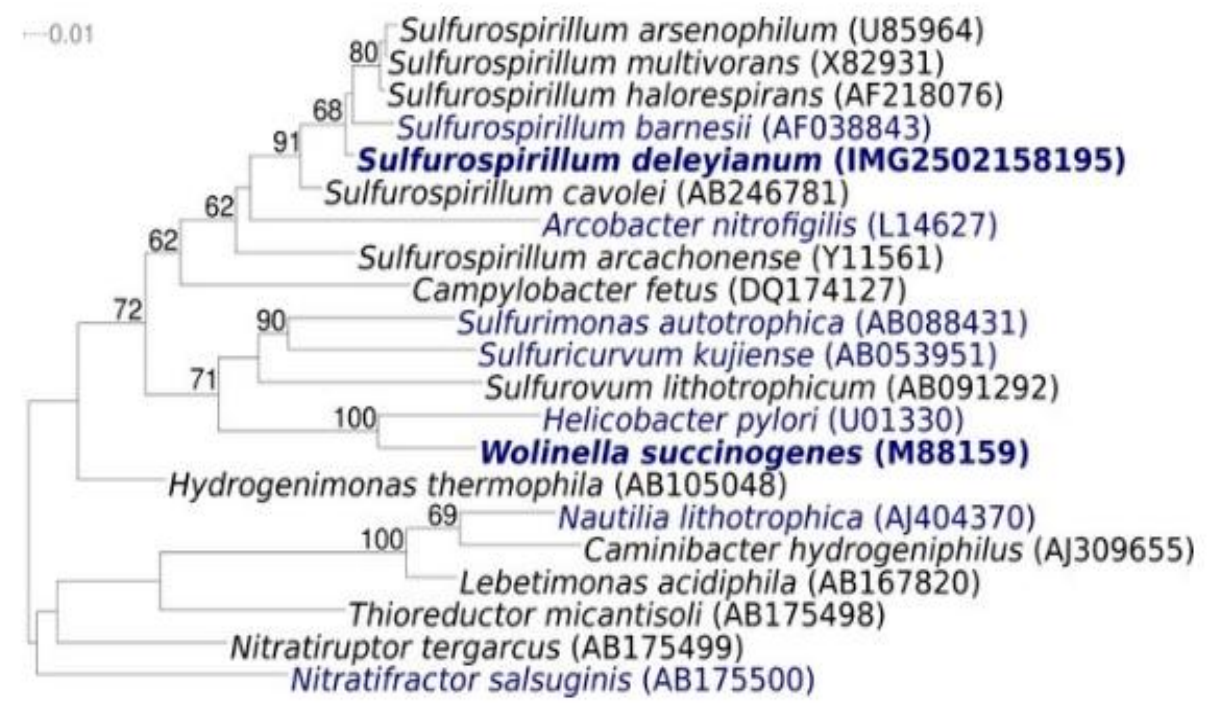

Fig. 5 Phylogenetic Tree Highlighting the Position of S.

\section{RESULTS AND DISCUSSIONS}

In this study, we first introduced the nucleus genome and the lateral genes of C. embryo, based on the closed genome of the seven C. embryo strains. After that, a novel phylogenetic analysis is used using the nucleus genome of $21 \mathrm{C}$. embryo strains with two sub-species mentioned above.

The results show that the genome of C. embryo subsp. venerealis $96 / 508$ has a circular chromosome dimension of 1876655 bp with a mean $\mathrm{G}+\mathrm{C}$ of $34.5 \%$ and contains two mega plasmids of $38.365 \mathrm{bp}$ and $39.685 \mathrm{bp}$ with $1657,365 \mathrm{bp}$ as 64.04 . The general characteristics of the applied genomes are demonstrated in the following table. The genomic characteristics of strains 23/293, 40-82 and 121-84 have been studied earlier.

Table1 General Features of the Assembled Genomes STARINIDATA

\begin{tabular}{|c|c|c|c|c|c|}
\hline Features & $\begin{array}{l}\text { 04/654i(thisistu } \\
\text { dy) }\end{array}$ & 96/508i(thisistudy) & $03 / 293$ & $82-40$ & $84-112$ \\
\hline Genome size & $1,657,365$ & $1,876,658$ & $1,652,063$ & $1,795,321$ & $1,926,886$ \\
\hline $\mathrm{G}+\mathrm{CiContenti} \%$ & 29.3 & 34.5 & 33.6 & 33.3 & 33.6 \\
\hline No.iofirRNAigenes & 3 & 6 & 3 & 3 & 3 \\
\hline No.iofitRNAigenes & 39 & 29 & 46 & 43 & 43 \\
\hline $\begin{array}{l}\text { No.iofihomopolymericiG+Citractsi }(>8 \\
\text { bp) }\end{array}$ & 33 & 31 & 32 & 30 & 34 \\
\hline No.iofiopenireadingiframes & 2,006 & 1,293 & 1,653 & 1,769 & 1,992 \\
\hline Plasmids & 1 & 2 & 1 & 0 & 1 \\
\hline Sizei(bp) & 26.365 & 26.03 & $91,398 / 33$ & 0 & 61,141 \\
\hline $\mathrm{G}+$ Cicontent $\%$ & 29.0 & 26.5 & $29.6 / 32.5 / 30.6$ & 0 & 31.5 \\
\hline Sapilocusitype & B & A & A & A & A \\
\hline Insertionielements & 0 & 1 & 13 & 0 & 5 \\
\hline Restrictioniorimodificationilocusitype & NA & I & I & NA & I \\
\hline CRISPR-casisystem & NA & NA & NA & yes & NA \\
\hline
\end{tabular}

Comparison of the seven genomes of closed mercury embryos (82-40, 84-112, 65/4, 96/508 and 03/293) showed highly synthesized genomes, > 89.6\% sequence, respectively. The main C. embryoiwas genome, defined on the IORFs contained in the closed genomes of the five C. embryo strains, has been identified to include $1,653,325 \mathrm{bp}$ and 1609 ORFs. The gene core was then identified in 16 additional embryonic strains and the genetic distance of all 21 major genomes was maximized with a phylogenetic probability tree. More pronounced differences between the five genomes of C. difficile embryos may occur in secondary genes.

Table 1 lists the identified SAP sites, insertion sequences, genomic islands, type I restriction systems, predictions and CRISPR-casi systems of 21 C. embryo. C. embryo strains analyzed included sap loci, but the composition of this region differed between species. Sap-type B and with genome B species, whereas SAP-type A strains were obtained in homogeneous clusters. The two C. embryo strains are completely kinetic, with a sequence identity of $92.9 \%$, and different features are not indistinguishable with DNA-DNA hybridization, which may arise that establish the validity of subgroup differentiation and preclude a taxonomic composition. Suitable for desired features. In addition, the reliability of the $2 \%$ glycine tolerance test can be influenced by the fact that glycine tolerance may be transmitted by phages.

Many molecular assays for theireconnaissance of C. embryo species have been discovered: however, none of the molecular assays fully correspond to the phenotypic identification of $\mathrm{C}$. embryo species. In a concurrent study, phylogenetic analysis of the nuclear genomes divides the original embryos into two clusters. All strains that are phenotypically derived as CFVs (such as biovar intermedius) assemble into a single genomic cluster of the nucleus, containing only strains with MLST ST-4 and incorporating IS elements and a system type Have RM.

The phenotypic isolates identified as CFF were divided into two clusters. Three strains of CFF, 03/293, Zaf 65, and ADRI 1362 were assigned to the main genome cluster with CFV and CFVI strains, despite the phenotype of CFF dioxins. The similarity of MLST identification and nucleus genome clustering can be explained 
by the fact that MLST is a small-scale reflection of the Icore genome. Phylogenetic analysis showed to have explicit similarity with MLST ST of the strains. The genetic distance between strains with the same STs is very low as the strains representing ST4 and ST2, and the genetic distance for strains with varying STs is reduced. Figure 7 and Table 2 illustrate the genome and accessory analysis of all embryonic species in this study.

The research also hypothesized that C. embryo could be an unknown member of the human gastrointestinal microbiota. To observe that if a healthy human could act as a natural reservoir of C. embryo, we compared all genomes (182) with the Human Pan-Microbial Association Database 28. This allows us to scan 7121 weapons-related metagenomic datasets that are internationally sampled from human feces. Surprisingly, C. 8 embryos were detected in 8 C. Samples from healthy humans. In contrast, Escherichia coli was detectable in 7\% of samples and human pathogens of C. jejuni and C. coli were detected in less than $0.5 \%$ of samples. Of the $10 \mathrm{C}$. fetal genotypes observed in the gastrointestinal microbiota of healthy individuals, $22.11 \%$ belonged to the new generation of bovine strains and $77.89 \%$ belonged to the human family strains (Figure 7). No relationship was found with gender, sex, age, ethnicity, or geographic origin of the donor or sample of origin. Similarly, abundance data showed no enrichment in other microbial organisms. Taken together, these data suggest that microbial community structure does not determine the success of embryo colonization. Interestingly, of the strains found from human families, TW2 represents $94 \%$ of the strains found in healthy unique, indicating a clonal expansion of this genotype in the human sample population (Figure 7). TW2 genotype belongs to cluster 5, which also enumerates CA24 genotype, which is responsible for the prevalence of C. embryo among men who have sex with men, with human-to-human transmission.

Campylobacter embryo transmission in humans is generally very uncommon, but often invasive and sometimes fatal. Campylobacter embryo transmission should be usually common in patients with nonspecific fever, those who may be at risk of occupation, or those who are immune to underlying or acquired, humoral, or cellular immunity due to underlying diseases, be suspicious, and be suspicious. There is no evidence to detect C. embryo in the stool of diarrhea patients, but laboratory diagnosis is performed with optional culture environment that inhibit C. embryo growth. A seroepidemiological study may complement these findings and correct for possible misdiagnosis, but this awaits the improvement of a embryo specific C. seroassay. Infection with Campylobacter embryos appears primarily shingles, with sheep and cattle as the main reservoirs. Direct contact with animals is a significant path, especially for some professions, such as farming or veterinary work. Our hypothesis is that humans are exposed to C. embryo through contaminated cattle and cattle products, especially the liver. Following this exposure, individuals are predominantly immune to the risk of clinical disease. Exposure evaluation studies await appropriate diagnosis of C. embryo in food. A systematic study of conditions samples using appropriate tools for molecular culture and culture provides significant information for assessing the environmental risks of human pollution. Human-to-human transmission of C. embryo to humans has been suggested to occur among very susceptible infants and encourage the implementation of precautionary measures.
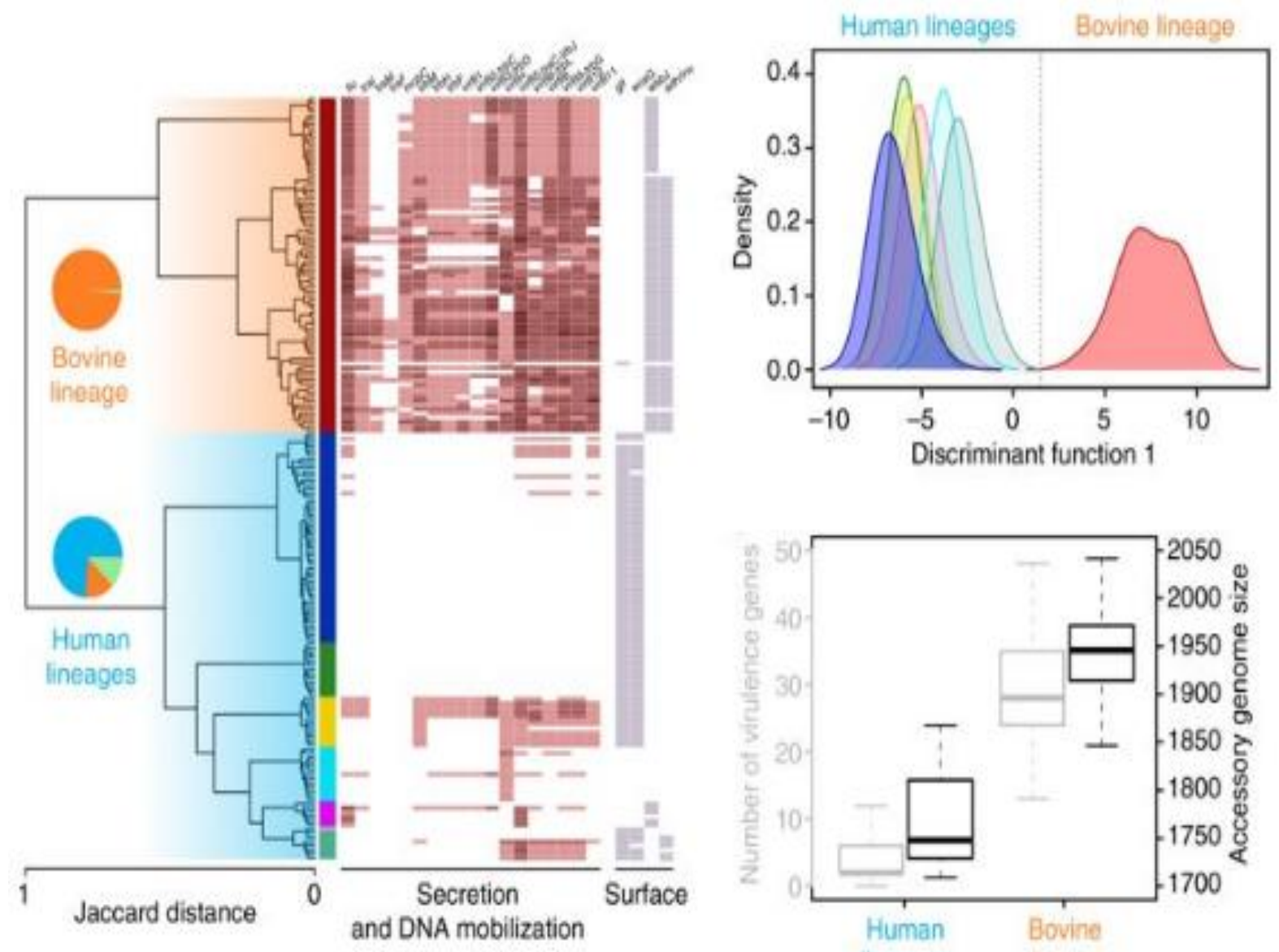

Fig. 6 Accessory Genome Analysis

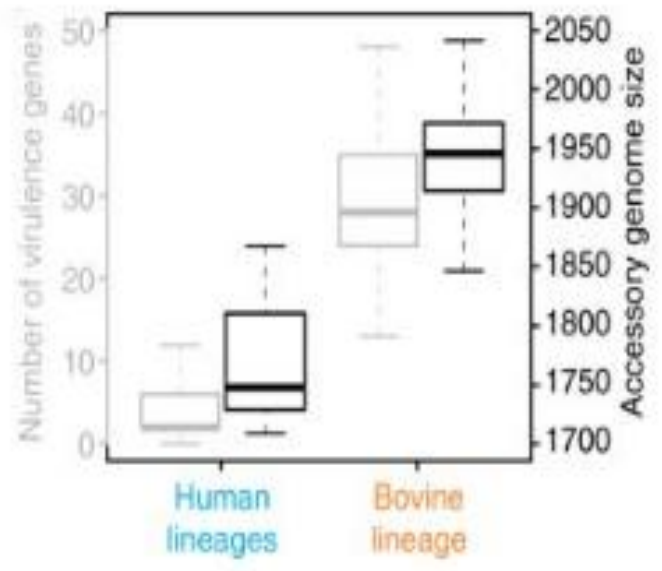

\section{CONCLUSIONS}

In this study, we introduced a phenotype of C. embriono subsp. The obtained CFVI strains phenotyped with CFV strains in cluster B belonging to the phylogenetic tree, while the secondary genes from the CFV and CFVI strains did not show a paradoxical tendency for a specific CFV or CFVI region. It is noteworthy that, with the exception of the ADRI 612 strain, all AFLPs introduced CFV markers and placed a promoter at the SAP locus, a prevention that is absent in most CFVI strains. All of the current predictive proteins are hypothesized to be functionally unknown, but one may suggest a prediction of a phenotype that is different, especially the H2S generation between CFV and CFVI strains.

The differentiation between C. embryoIsubspecies moves more than taxonomic interest. Clinically, subspecies have been introduced in a way that should be different. CFV (including biofuels intermediate) has been identified as the causative agent of bovine genital Campylobacteriosis (BGC). There is a generally accepted association between C. embryo species and their specific diagnostic physiology, epidemiological features, and host niche characteristics. Cattle products may be tested against CFV deficiency as listed in the World Animal Health Standard (OIE). When detected in such screening for C. embryo, subspecies identification is generally performed by phenotyping, as described in the OIE Manual of Diagnostic Tests and TerrestrialIAnimals Vaccines (18). However, there is no evidence for phenotypic markers (glycine tolerance and $\mathrm{H} 2 \mathrm{~S}$ production) that are associated with the robustness profile of C. embryo species. Subsequent 
diagnoses of C. embryo should preferably detect genomic features isolated with different host ducts and nests. However, the genes introduced in this paper are not consistently produced in the CFV genome and are therefore useless as a diagnostic method. Expecting the absence of skin-related genes, it can be recognized that the current relationship between phenotype and agriculture is questionable, since several phenotypically defined CFF strains have similar characteristics to CFV species, based on the genome. Core and similarity are accessories, as shown in this study. Failure to master phenotypes and genomic characteristics of C. embryo species encourages critical evaluation of the clinical significance of C. fetal subs pieces using phenotypic methods.

Table 2 General Characteristics of $C$. Embryo Strains

\begin{tabular}{|c|c|c|c|c|c|c|c|c|c|c|c|c|c|c|}
\hline \multirow[b]{2}{*}{ Strain } & \multirow[b]{2}{*}{ Country } & \multirow[b]{2}{*}{ Source } & \multicolumn{3}{|c|}{ Phenotype } & \multicolumn{2}{|c|}{ Genotype } & \multirow[b]{2}{*}{$\begin{array}{l}\text { Core } \\
\text { genome } \\
\text { cluster }\end{array}$} & \multicolumn{6}{|c|}{ Accessory genes } \\
\hline & & & $\begin{array}{c}1 \% \\
\text { Glycine } \\
\text { tolerance }\end{array}$ & $\begin{array}{c}\mathrm{H}_{2} \mathrm{~S} \\
\text { produc } \\
\text { tion }\end{array}$ & $\begin{array}{l}\text { Pheno } \\
\text {-typic } \\
\text { ID }\end{array}$ & AFLP & $\begin{array}{c}\text { MLST } \\
\text { (ST) }\end{array}$ & & $\begin{array}{l}\text { sap- } \\
\text { type }\end{array}$ & $\begin{array}{l}\text { Prophage } \\
\text { in sap- } \\
\text { locus }\end{array}$ & $\begin{array}{c}\text { GI } \\
\text { with } \\
\text { T4SS }\end{array}$ & IS & $\begin{array}{c}\text { type I } \\
\text { RM } \\
\text { system }\end{array}$ & $\begin{array}{c}\text { CRISPR } \\
\text {-cas }\end{array}$ \\
\hline $82-40$ & US & Human & + & + & Cff & $\mathrm{Cff}$ & 6 & B & A & - & - & - & $+/-$ & + \\
\hline $110800-21-2$ & $\mathrm{NL}$ & Bovine (bull) & + & + & Cff & Cff & 2 & B & A & - & + & - & $+/-$ & - \\
\hline BT 10/98 & UK & Ovine & + & + & Cff & Cff & 2 & B & A & - & - & - & $+1-$ & - \\
\hline $04 / 554$ & AR & Bovine (foetus) & + & + & Cff & Cff & 5 & B & B & - & - & - & - & - \\
\hline $98 / v 445$ & UK & Bovine (bull) & + & + & Cff & Cff & 3 & B & B & - & + & - & - & + \\
\hline $03 / 293$ & AR & Bovine (foetus) & + & + & Cff & Cfvi & 4 & $\mathbf{A}$ & A & - & + & + & + & - \\
\hline ADRI 1362 & AR & Bovine & + & + & Cff & Cfvi & 4 & $\mathbf{A}$ & A & + & + & + & + & - \\
\hline Zaf 65 & SA & Bovine & + & + & Cff & Cfvi & 4 & $\mathbf{A}$ & A & - & - & + & + & - \\
\hline $01 / 165$ & AR & Bovine (mucus) & - & + & Cfvi & Cfvi & 4 & $\mathbf{A}$ & A & - & + & + & + & - \\
\hline $02 / 298$ & AR & Bovine (foetus) & - & + & Cfvi & Cfvi & 4 & $\mathbf{A}$ & A & - & + & + & + & - \\
\hline $03 / 596$ & AR & Bovine (foetus) & - & + & Cfvi & Cfvi & 4 & $\mathbf{A}$ & A & - & + & + & + & - \\
\hline $92 / 203$ & AR & Bovine (placenta) & - & + & Cfvi & Cfvi & 4 & $\mathbf{A}$ & A & - & + & + & + & - \\
\hline $97 / 532$ & AR & Bovine (mucus) & - & + & Cfvi & Cfvi & 4 & $\mathbf{A}$ & A & - & + & + & + & - \\
\hline $98 / 25$ & AR & Bovine (foetus) & - & + & Cfvi & Cfvi & 4 & $\mathbf{A}$ & A & - & + & + & + & - \\
\hline WBT 011/09 & UK & unknown & - & + & Cfvi & Cfvi & 4 & $\mathbf{A}$ & A & - & + & + & + & - \\
\hline Zaf 3 & SA & Bovine (foetus) & - & + & Cfvi & Cfvi & 4 & $\mathbf{A}$ & A & - & - & + & + & - \\
\hline ADRI 513 & AU & unknown & - & + & Cfvi & $\mathrm{Cfv}$ & 4 & A & A & - & - & + & + & - \\
\hline CCUG 33872 & $\mathrm{CZ}$ & unknown & - & + & Cfvi & Cfv & 4 & $\mathbf{A}$ & A & + & - & + & + & - \\
\hline $84-112$ & US & Bovine & - & - & $\mathrm{Cfv}$ & Cfv & 4 & $\mathbf{A}$ & A & + & + & + & + & - \\
\hline $97 / 608$ & AR & Bovine (placenta) & - & - & $\mathrm{Cfv}$ & Cfv & 4 & $\mathbf{A}$ & A & + & + & + & + & - \\
\hline B10 & US & Bovine & - & - & $\mathrm{Cfv}$ & Cfv & 4 & A & A & + & + & + & + & - \\
\hline CCUG 33900 & $\mathrm{~F}$ & Bovine (abortion) & - & - & $\mathrm{Cfv}$ & Cfv & 4 & $\mathbf{A}$ & A & + & + & + & + & - \\
\hline LMG 6570 & $\mathrm{BE}$ & Bovine & - & - & $\mathrm{Cfv}$ & Cfv & 4 & A & A & + & + & + & + & - \\
\hline
\end{tabular}




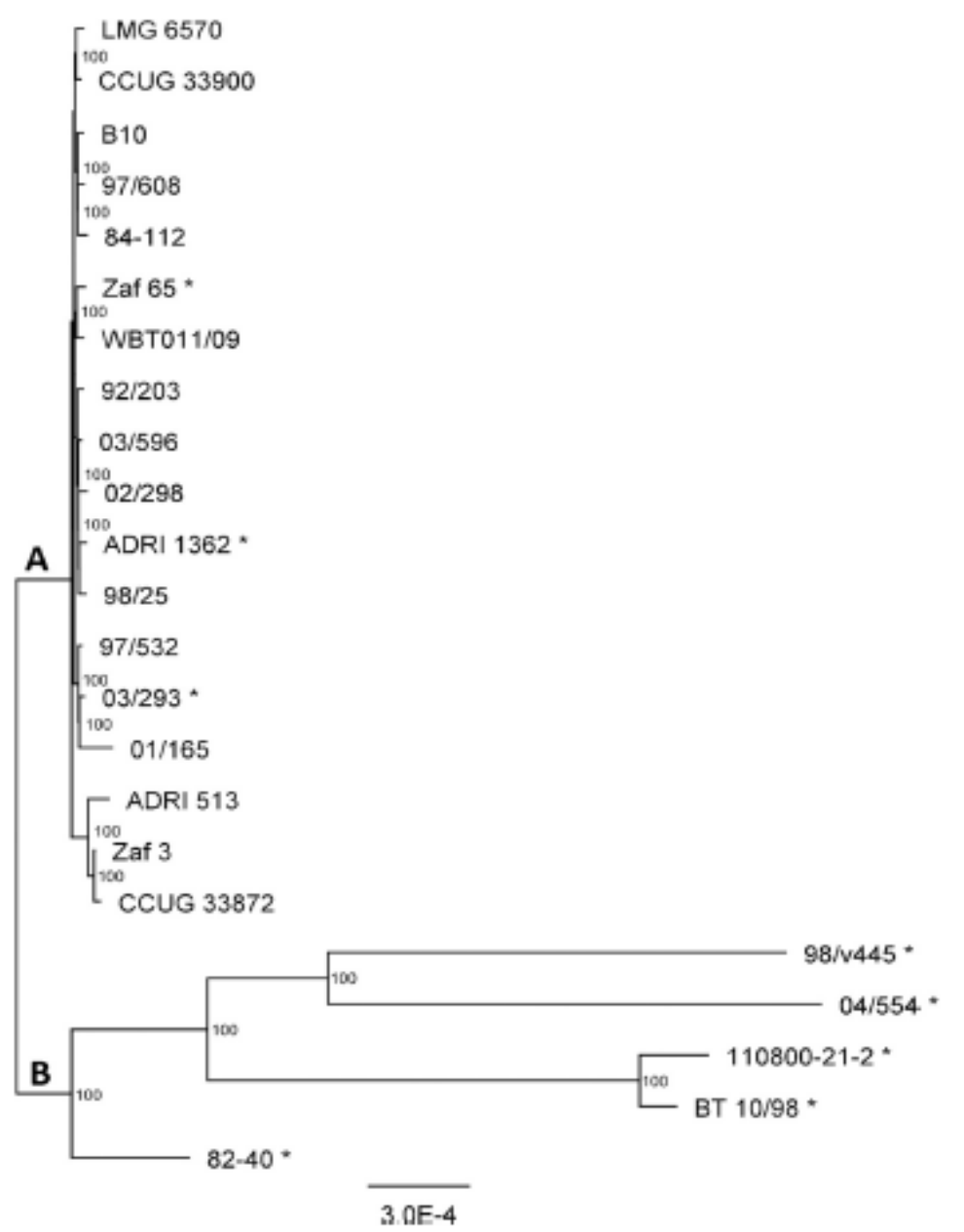

Fig. 7 PhylogeneticiTree of $C$. embryo StrainsIbased on the Core Genomes. Strains that are Phenotypically Identified as $C$. embryo subsp. Embryo (CFF) are Marked with anIAsterisk. Bootstrap Supportsiare Indicated on the Branches. The ScaleiRepresents the MeaniNumber of Nucleotide Substitutions per site.

\section{REFERENCES}

[1]. Ali A, Soares SC, Santos AR, Guimaraes LC, Barbosa E, et al. 2012. Campylobacter embryo subspecies: Comparative genomics and prediction of potential virulence targets. Gene.

[2]. Bergen MA, Linnane S, van Putten JP, Wagenaar JA, 2005. Global detection and identification of Campylobacter embryo subsp. venerealis. Rev Sci Tech 24: 1017-1026.

[3]. Blaser MJ, 1998. Campylobacter embryo -emerging infection and model system for bacterial pathogenesis at mucosal surfaces. Clin Infect Dis 27: 256-258.

[4]. Chang W, Ogg JE., 1971. Transduction and mutation to glycine tolerance in Vibrio embryo . Am. J. Vet. Res. 32:649-653.

[5]. Fouts DE, Mongodin EF, Mandrell RE, Miller WG, Rasko DA, et al., 2005. Major structural differences and novel potential virulence mechanisms from the genomes of multiple Campylobacter species. PLoS Biol $372-85$.

[6]. Garcillan-Barcia MP, Francia MV, de la Cruz F., 2009. The diversity of conjugative relaxases and its application in plasmid classification. FEMS Microbiol Rev 33: 657-687

[7]. Gonzalez JM, Xu H, Ofori E, Elovitz MA, 2007. Toll-like receptors in the uterus, cervix, and placenta: is pregnancy an immunosuppressed state? Am J Obstet Gynecol 197: 296 e291-296.

[8]. Iraola G, Perez R, Naya H, Paolicchi F, Harris D, et al., 2013. Complete Genome Sequence of Campylobacter embryo subsp. venerealis Biovar Intermedius, Isolated from the Prepuce of a Bull. Genome Announc 1.

[9]. Jansen R, Embden JD, Gaastra W, Schouls LM., 2002. Identification of genes that are associated with DNA repeats in prokaryotes. Mol Microbiol. 43:1565-1575.

[10]. Jay-Russell MT, Bates A, Harden L, Miller WG,Mandrell RE., 2012. Isolation of Campylobacter fromferal swine (Sus scrofa) on the ranch associated with the 2006 Escherichia coli O157:H7 spinach outbreak investigation in California. Zoonoses Public Health 59:314-319.

[11]. Lee CA, Babic A, Grossman AD, 2010. Autonomous plasmid-like replication of a conjugative transposon. Mol Microbiol 75: 268279.

[12]. Man SM (2011) The clinical importance of emerging Campylobacter species. Nat Rev Gastroenterol Hepatol 8: 669-685.

[13]. McCallum M, Shaw GS, Creuzenet C, 2011. Characterization of the dehydratase WcbK and the reductase WcaG involved in GDP-6deoxymanno- heptose biosynthesis in Campylobacter jejuni. Biochem J 439: 235-248.

[14]. Poele EM, Bolhuis H, Dijkhuizen L, 2008. Actinomycete integrative and conjugative elements. Antonie Van Leeuwenhoek 94: 127143. 
[15]. Ryan A, Lynch M, Smith SM, Amu S, Nel HJ, et al., 2011. A role for TLR4 in Clostridium difficile infection and the recognition of surface layer proteins. PLoS Pathog 7: e1002076.

[16]. Skirrow MB, Blaser MJ, 2000. Clinical aspects of Campylobacter infections. In: Nachamkin I, Blaser, M J., editor. Campylobacter, 2nd Ed. Washington, D. C.: American Society for Microbiology. 69-88.

[17]. Trinchieri G, Sher A, 2007. Cooperation of Toll-like receptor signals in innate immune defence. Nat Rev Immunol 7: 179-190.

[18]. Tu ZC, Dewhirst FE, Blaser MJ, 2001. Evidence that the Campylobacter embryo sap locus is an ancient genomic constituent with origins before mammals and reptiles diverged. Infect Immun 69: 2237-2244.

[19]. Tu ZC, Eisner W, Kreiswirth BN, Blaser MJ, 2005. Genetic divergence of Campylobacter embryo strains of mammal and reptile origins. J Clin Microbiol 43: 3334-3340.

[20]. Wagenaar JA, van Bergen MAP, Newell DG, Grogono-Thomas R, Duim B., 2001. Comparative study using amplified fragment length polymorphism fingerprinting, PCR genotyping, and phenotyping to differentiate Campylobacter embryo strains isolated from animals. J. Clin. Microbiol. 39: 2283-2286. http://dx.doi.org/10.1128/JCM.39.6.2283-2286.2001. 